\title{
Evaluation and correlation of mammographically suspicious lesions with histopathology at Addington Hospital, Durban
}

\author{
B D Cupido, F Vawda, A Sabri, C T Sikwila \\ Department of Radiology, Addington Hospital, Durban, South Africa \\ B D Cupido, MB ChB, FCRad (Diag) SA
}

Department of Radiology, School of Surgical Disciplines, Nelson R Mandela School of Medicine, College of Health Sciences, University of KwaZuluNatal, Durban, South Africa

F Vawda, MB ChB, FCRad (Diag) SA

Cypress Health Region Hospital, Department of Radiology, Saskatchewan, Canada

A Sabri, MD, MMed, FCRad (Diag) SA

University Teaching Hospital, Lusaka, Zambia

C T Sikwila, MB ChB, FCRad (Diag) SA

Corresponding author: B D Cupido (brindleycupido@yahoo.com)

\begin{abstract}
Background. Stereotactic core-needle biopsies (SCNBs) are a reliable alternative to surgical biopsy for microcalcifications. The positive predictive value (PPV) of SCNB has been shown to be reproducible in several studies using the Breast Imaging Reporting and Data System (BIRADS) classification, which is the current gold standard in mammographic reporting. At this stage, no study has been done in KwaZuluNatal to assess local outcomes against BIRADS. The current standard of care utilises vacuum-assisted breast biopsy, but is not available in a resource-constrained environment such as ours. The need, therefore, is for constant evaluation of existing practice to ensure that it is optimised for the challenges and limitations facing local radiologists.

Objective. To assess the PPV of SCNB in Addington Hospital, and to compare it with that of BIRADS.

Material and methods. Mammographically detected lesions were assigned to 3 categories: benign, indeterminate and suspicious. A retrospective review of 67 SCNBs was performed for lesions falling within the suspicious category, and the PPV and rates of ductal carcinoma in situ (DCIS) were determined.

Results. Our study demonstrated a PPV of 20.9\%. This correlated well with international studies for BIRADS 4 and 5 lesions. DCIS accounted for $21.4 \%$ of detected malignancies, which is in keeping with current literature.

Conclusion. Despite resource limitations, local outcomes were comparable with those of BIRADS. Given our fairly general categorisation of lesions, however, it should be emphasised that BIRADS allows better organisation, consistency and clarity in breast imaging reporting, as well as accurate data comparison between centres facing limitations similar to our own.

S Afr Med J 2013;103(4):251-254. DOI:10.7196/SAMJ.6361
\end{abstract}

The Breast Imaging Reporting and Data System (BIRADS) Lexicon was developed in 1985 and is currently in its 4th edition. Its introduction acknowledged the need for a standardised system of reporting and risk stratification, to aid in predicting the probability of malignancy in relation to mammographically identified lesions. To date, it has proven to be an accurate predictor of malignancy, depending on 
the category to which mammographic lesions are assigned. As such, BIRADS has been adopted by a large number of mammography centres throughout the world.

However, many centres in South Africa (SA), including ours, have been slow to embrace BIRADS, even though it is part of the standard curriculum in all universities. The reasons for this are not completely clear, but a common view is that BIRADS is limited in advising how descriptive findings used in the BIRADS Lexicon should be linked to the final BIRADS assessment categories. ${ }^{[1]}$ Furthermore, data are limited on the prospective classification of microcalcifications according to the lexicon. ${ }^{[1]}$

Addington Hospital typically performs 1500 - 2000 mammography examinations per year. Until early 2011, the mammography team consisted of a part-time radiologist and 2 full-time mammographyaccredited radiographers. Owing to limited resources and long waiting lists, our focus has been on high-risk patients and diagnostic mammography in symptomatic patients. Obtaining stereotactic biopsies is time-consuming and limits the number of examinations that can be scheduled per day, thus increasing patient waiting times for mammography. An assessment of our current practice was therefore deemed necessary to ascertain whether we are performing enough biopsies as benchmarked against current international standards, and whether our results warranted increased patient waiting times. Furthermore, we thought it imperative to ascertain the impact on standard of care of not using BIRADS.

The classic signs of malignancy are spiculated masses and linear/ branching calcifications. However, less than $20 \%$ of non-palpable breast cancers present as a spiculated mass. ${ }^{[2]}$ Subtle signs include focal architectural distortion, other calcifications, developing densities, single dilated ducts and asymmetric areas of increased density when compared with the contralateral breast. Notably, up to one-third of non-palpable breast cancers can present with calcifications alone, ${ }^{[2]}$ which further highlights the importance of accurate analysis of microcalcification patterns.

The above descriptors are generally agreed to be the most accurate predictors of malignancy, and our unit uses them as standard descriptors of lesions. Whereas BIRADS allows fairly accurate stratification of patients into risk categories, our unit typically uses 3 categories that determine the follow-up management strategy: typically benign/normal, indeterminate and suspicious. Typically benign/ normal mammograms receive routine follow-up mammograms. The indeterminate category consists of those lesions which lack suspicious features, but also lack the characteristic benign features/proven stability needed to be assigned to the benign/normal category; these lesions receive short-term (6-month) mammographic follow up. Mammograms deemed suspicious are typically considered for stereotactic or ultrasound-guided biopsies.

The primary purpose of this study was to use our current approach to assess the correlation between pre-biopsy classification of non-palpable mammographically suspicious lesions, and their underlying histology, and to compare our positive predictive value with existing published data based on BIRADS.

\section{Material and methods Study design and patients}

We retrospectively reviewed stereotactic core-needle biopsies (SCNB) performed at Addington Hospital for non-palpable lesions. Only lesions not visualised at ultrasound examination were referred for stereotactic biopsies. The time period of our review was January 2007 - June 2011. The study was reviewed and approved by the Biomedical Research and Ethics Committee of the University of KwaZulu-Natal, while institutional approval was granted by the Addington Hospital Ethics Committee.

All mammogram interpretations and biopsies were performed by the same highly experienced radiologist (with more than 30 years in this sub-speciality).

The BIRADS categories were not utilised in the reporting of these images. All lesions regarded as 'suspicious' were biopsied and would, had BIRADS been utilised, in all likelihood have corresponded to a BIRADS 4, or higher, category.

\section{Imaging and evaluation}

All mammographic examinations were performed on a Performa Stereo unit by GE Healthcare utilising a screen-film technique. Biopsy samples were obtained using a Bard Magnum Core Biopsy Instrument with a 14-gauge, $13 \mathrm{~cm}$ needle. Specimen radiography was not performed on any of the samples as this facility was not available. Instead, a focal mammographic exposure of the area of concern was performed at the time of biopsy procedure, using a highly collimated beam to assess the lesion following removal of the relevant lesions/ microcalcifications.

Positive mammograms were considered to be those with a positive stereotactic biopsy result for carcinoma or with atypical ductal hyperplasia. The positive predictive value (PPV) was defined as the number of malignant biopsy results divided by the total number of biopsies performed.

\section{Results}

The women in the study were $40-88$ years of age (mean age: 59.3 years \pm 11.6 ). A total of 67 SCNBs were performed during the specified period. The 67 lesions comprised 33 (49.3\%) masses, $31(46.3 \%)$ microcalcifications, $1(1.5 \%)$ asymmetry and $2(3 \%)$ developing densities (Fig. 1).

Histological testing of SCNB specimens demonstrated malignancy in $14(20.9 \%)$ biopsies, with $46(68.7 \%)$ specimens showing benignancy. In 7 (10.4\%) specimens, the pathology report stated benign features but questioned whether the specimens were of a representative nature (Fig. 2.). Of these 7 patients, 2 underwent excisional biopsies, demonstrating an invasive lobular carcinoma in 1 , and an intraductal papilloma in the other. Of the remaining 5 cases, 3 were lost to follow-up while the remaining 2 had followup mammograms. Of the 14 malignancies, $9(64.3 \%)$ presented as masses/nodules, $3(21.4 \%)$ as microcalcifications, $1(7.1 \%)$ as an asymmetry and $1(7.1 \%)$ as a developing density.

DCIS was diagnosed in 3 (21.4\%) SCNBs with malignant histology, with invasive ductal carcinoma accounting for 11 (78.6\%). Results of excisional biopsies showed 1 case of invasive lobular carcinoma and 1 intraductal papilloma.

\section{Discussion}

Despite mammography's high sensitivity $(70-90 \%)$ for the early detection of breast cancer, its efficacy is limited by rather poor PPV. ${ }^{[2]}$ As a result, stereotactic biopsy has become an indispensable tool in the management of mammographically suspicious lesions. Although vacuum-assisted breast biopsy is currently considered the standard of care in First World settings - particularly for microcalcifications - studies have shown SCNB to be highly accurate when used appropriately. ${ }^{[3]}$

From our study, we determined the PPV for suspicious lesions to be $20.9 \%$. This finding is similar to that of Mendez et al., who demonstrated a PPV of $20 \%$ for BIRADS 4 and 5 lesions for breast cancer or atypia. ${ }^{[4]}$ 


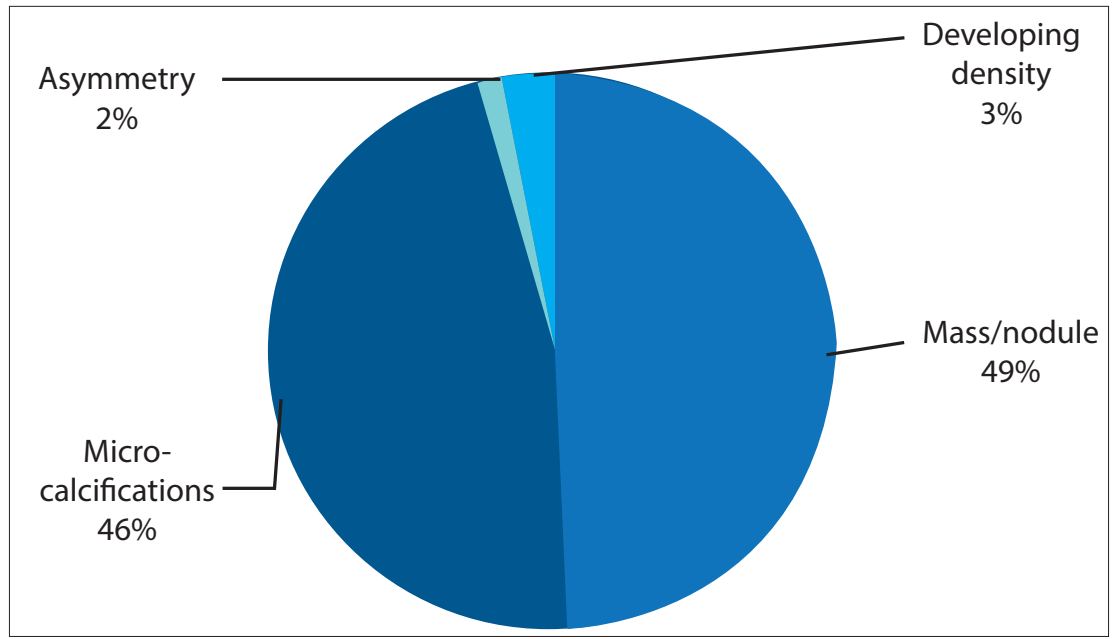

Fig. 1. The women were $40-88$ years of age (mean age: 59.3 years \pm 11.6 SD). A total of 67 SCNB's were performed during the specified period. The 67 lesions comprised 33 (49.3\%) masses, 31 (46.3\%) microcalcifications, 1 (1.5\%) asymmetry and 2 (3\%) developing densities.

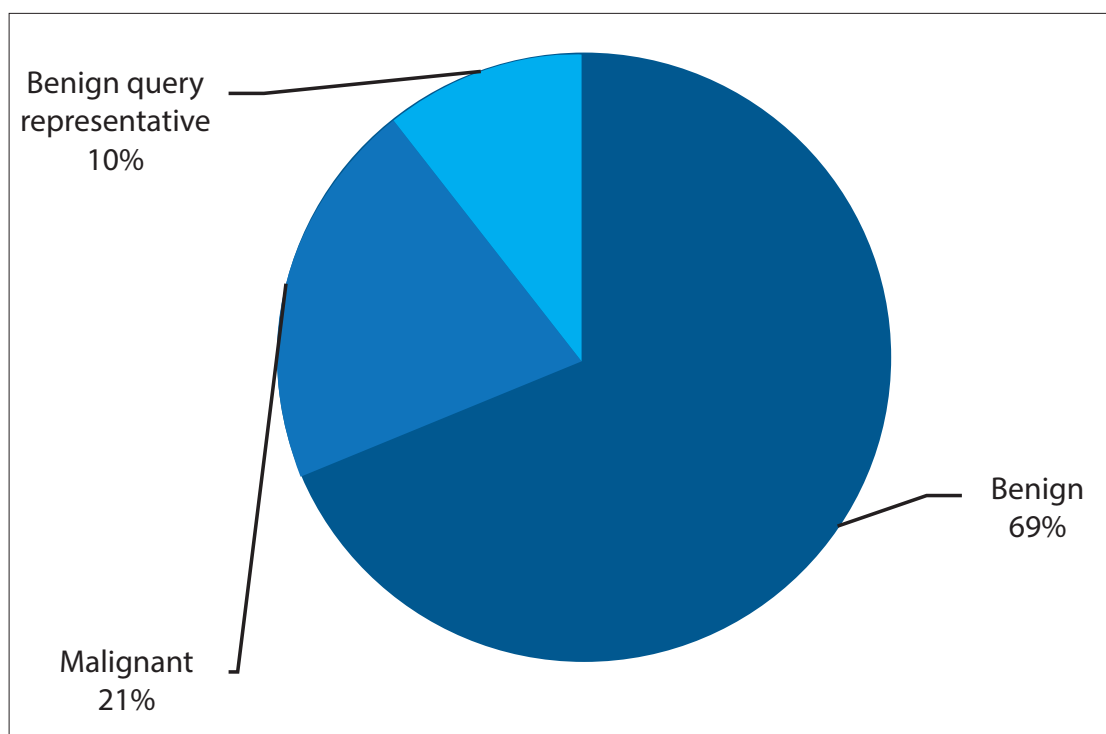

Fig. 2. DCIS was diagnosed in 3 (21.4\%) SCNBs with malignant histology, with invasive ductal carcinoma accounting for 11 (78.6\%). Results of excisional biopsies showed 1 case of invasive lobular carcinoma and 1 intraductal papilloma

Lazarus et al. ${ }^{[5]}$ conclude that subcategorisation of suspicious lesions into $4 \mathrm{a}, 4 \mathrm{~b}$ and $4 \mathrm{c}$ is helpful in predicting the likelihood of malignancy. In our unit no subcategories were assigned, so further risk stratification was not possible. Though the PPV of our study was comparable with the PPV for combined BIRADS 4 and 5 lesions, ${ }^{[4]}$ our lack of categorisation is less helpful in terms of communicating pre-biopsy malignancy risk to referring physicians, patients, etc.

Roth et al..$^{[3]}$ recommend that specimen radiography should be performed for all stereotactic biopsy specimens, to ensure that representative samples of microcalcifications are obtained. In our unit, however, specimen
2 broad subtypes: ductal and lobular carcinoma. Invasive ductal carcinoma accounts for up to $85 \%$ of invasive cancers, with lobular carcinoma comprising about $10-15 \%$ of all invasive cancers. ${ }^{[6]}$

Ductal carcinoma in situ (DCIS), which is a precursor to invasive ductal carcinoma, represents $20-25 \%$ of malignancy detected at mammographic screening. Because most DCIS are high-grade, it is recommended that suspicious microcalcifications be aggressively investigated. ${ }^{[7]}$ Furthermore, a strong correlation between rates of detection of DCIS and rates of detection of small invasive cancers at screen-film mammography has been demonstrated. ${ }^{[8]}$ In our study, DCIS accounted for $21.4 \%$ of malignancies identified on SCNB specimens, in keeping with current literature. ${ }^{[7]}$

Proliferative lesions without atypia, such as ductal hyperplasia, carry only a slightly increased risk of malignancy. Atypical ductal hyperplasia, however, is known to have a moderate risk of invasive breast cancer, ${ }^{[9]}$ and thus carries with it an indication for excisional biopsy. None of our biopsies revealed atypical ductal hyperplasia.

Two patients in our sample underwent excisional biopsies for suspicious nodules, which returned a diagnosis of invasive lobular carcinoma in one and a diagnosis of an intraductal papilloma in the other. The case of invasive lobular carcinoma is particularly interesting because initial SCNB demonstrated benign findings, although the representative nature of the specimen was queried. The patient had a routine 6-month, 12-month and 24-month mammographic follow up, which demonstrated stability of the nodule that, in line with current recommendations, would indicate benignity. However, a mammogram performed at 36 months demonstrated a slight increase in size, prompting an excisional biopsy that demonstrated invasive lobular carcinoma. This finding represented the one false negative, confirmed by excision biopsy, in the study. The actual false-negative rate for SCNB is not known because most studies in the literature have not provided the rigorous long-term follow-up (at least 2 - 3 years) required to identify all false-negative diagnoses. $^{[10]}$

Of the other 5 cases falling into the nonspecific benign category, 3 were lost to follow-up. The remaining 2 had mammographic follow- ups for microcalcifications at 6,12 and 24 months with no interval change. This frequency is in keeping with the recommendation by Lee et al. of 6-month follow up for 
cases that yield nonspecific benign results at SCNB, and yearly screening mammography for cases with specific benign results. ${ }^{[1]}$ An alternative to continued mammographic surveillance would have been a repeat SCNB with an 11-gauge, vacuum-assisted device, but this was not available.

\section{Limitations}

Our study was limited by our SCNB sampling procedure, which did not utilise specimen radiography as recommended by Roth et al. ${ }^{3}$ For logistical reasons, we performed a repeat focal mammographic view to assess biopsy of relevant lesions. This approach is clearly suboptimal as it increases patient exposure during the examination. It also fails to accurately comment on the representative nature of microcalcification specimens in particular, for which specimen radiography is mandatory. However, this has proven to be the only viable option in view of resource limitations: at present, performing specimen radiography would require either a second mammography unit, a cabinet X-ray system for point-of-care specimen radiography or an interruption in the procedure to allow for a specimen radiograph to be taken. Given our financial and time constraints, none of these options was viable at the time.

A further limitation was that all mammograms were read by a single reader, albeit one with 30 years' experience. The data are therefore not necessarily generalisable to everyday practice, especially where relatively junior radiologists are concerned. This further emphasises the need for a more universal system, such as BIRADS, which addresses these factors.

The data set of 67 biopsies was relatively small. A larger sample would definitely add greater statistical value to the findings, although the current results in addition to the literature, do provide a good reference point for future modifications in our unit.

A final limitation was the lack of rigorous follow-up in those patients with benign histology on core-needle biopsy. As follow-up in our local setting is and will continue to be a major challenge, the incidence of non-benign lesions missed on initial SCNB remains an ongoing concern at present and into the foreseeable future.

\section{Conclusion}

The absence of subcategorisation in the system used in our unit had no detrimental effect on definitive patient management, as our PPV was comparable with studies employing BIRADS. This consistency demonstrates that optimal outcomes can be achieved despite resource constraints, which are becoming increasingly prevalent.

A significant shortfall of our approach is its lack of the standard language and action-oriented approach to breast imaging reporting, which is one of the primary goals of BIRADS. The BIRADS Lexicon was specifically devised to ensure that commonly used terms such as 'pleomorphic', 'coarse heterogeneous' and 'spiculated' would have the same meaning regardless of the radiologist interpreting the study. The final assessment categories were also developed to ensure a conclusive and clear plan of action.

Although our overall outcomes were relatively similar to BIRADS, failure to adopt the latter could isolate a breast centre from mainstream mammography practice and compromise its ability to meaningfully engage other role players on a common platform. Therefore, it is highly recommended that BIRADS not only be taught but also practised.

Acknowledgements. We gratefully acknowledge Dr I J Movson for his role in the interpretation of mammograms used in our study. We also thank Dr D Ramaema for her invaluable input. Lastly, our utmost thanks to nurse M Ebrahim for her assistance with data collection.

\section{References}

1. Müller-Schimpfle M, Wersebe A, Xydeas T, et al. Microcalcifications of the breast: How does radiologic classification correlate with histology? Acta Radiol 2005;46:774-781.

2. Sickles EA. Mammographic features of 300 consecutive non-palpable breast cancers. Am J Roentgeno 1986;146(4):661-663

Roth WD, Von Smitten K, Heikkilä P, Edgren J, Laasonen L. Automated stereotactic core-needle biopsy of microcalcifications with correlation to surgical biopsy. Acta Radiol 1999;40:390-393.

4. Mendez A, Cabanillas F, Echenique M, Malekshamran K, Perez I, Ramos E. Mammographic features and correlation with biopsy findings using 11-gauge stereotactic vacuum-assisted breast biopsy (SVABB). Ann Oncol 2003;14:450-454. [http://dx.doi.org/10.1093/annonc/mdh088]

5. Lopez K, Basset LW. Invasive lobular carcinoma of the breast: Spectrum of mammographic, US and MR imaging findings. Radiographics 2009;29:165-176. [http://dx.doi.org/10.1148/rg.291085100]

6. Lazarus E, Mainiero MB, Schepps B, Koelliker SL, Livingston LS. BI-RADS Lexicon for US and Lazarus E, Mainiero MB, Schepps B, Koelliker SL, Livingston LS. BI-RADS Lexicon for US and mammography: Interobserver variability and positive

7. Evans AJ, Pinder SE, Ellis IO, Wilson AR. Screen detected ductal carcinoma in situ (DCIS) Overdiagnosis or an obligate precursor of invasive disease? J Med Screen 2001;8:149-151.

8. Weigel S, Decker T, Korsching E, et al. Calcifications in digital mammographic screening: Improvement of early detection of invasive breast cancers? Radiology 2010;255(3):738-745. [http:// Improvement of early detection of in

Sanders MA, Roland L, Sahoo S. Clinical implications of subcategorizing BI-RADS 4 breast lesions . Sanders MA, Roland L, Sahoo S. Clinical implications of subcategorizing BI-RADS 4 breast lesions
associated with microcalcification: A radiology-pathology correlation study. Breast J 2010;16(1):28-31 associated with microcalcification: A radiology-pathology correlation study. Breast J 2010;16(1):28-31,
Bassett L, Winchester DP, Caplan RB, et al. Stereotactic core-needle biopsy of the breast: A report of the joint task force of the American College of Radiology, American College of Surgeons and College of American Pathologists. CA Cancer J Clin 1997;47(3):171-190.

11. Lee CH, Philpotts LE, Horvath LJ, Tocino, I. Follow up of breast lesions diagnosed as benign with stereotactic core-needle biopsy: Frequency of mammographic change and false negative rate. Radiology 1999;212:189-194.

Accepted 19 November 2012 